\title{
The compressibility of rotating black holes in $D$-dimensions
}

\author{
Brian P. Dolan \\ Department of Mathematical Physics, National University of Ireland, \\ Maynooth, Ireland \\ and \\ Dublin Institute for Advanced Studies, 10 Burlington Rd., Dublin, Ireland \\ e-mail: bdolan@thphys.nuim.ie
}

July 31, 2018 


\begin{abstract}
Treating the cosmological constant as a pressure, in the context of black hole thermodynamics, a thermodynamic volume for the black hole can be defined as being the thermodynamic variable conjugate to the pressure, in the sense of a Legendre transform. The thermodynamic volume is explicitly calculated, as the Legendre transform of the pressure in the enthalpy, for a rotating asymptotically anti-de Sitter Myers-Perry black hole in $D$ spacetime dimensions. The volume obtained is shown to agree with previous calculations using the Smarr relation. The compressibility is calculated and shown to be non-negative and bounded.

Taking the limit of zero cosmological constant, the compressibility of a rotating black hole in asymptotically flat space-times is determined and the corresponding speed of sound computed. The latter is bounded above and has an elegant expression purely in terms of the angular momenta, in the form of quartic and quadratic Casimirs of the rotation group, $S O(D-1)$.
\end{abstract}

Report No. DIAS-STP-13-08

PACS nos: 04.60.-m; 04.70.Dy 


\section{Introduction}

The thermodynamics of black holes has been an active area of research ever since Bekenstein and Hawking's seminal papers on the entropy and temperature associated with the event horizon of a black hole, [1, 2]. Recently the rôle of pressure and volume has come under scrutiny in this context. It was pointed out in [3] that the presence of a cosmological constant, $\Lambda$, spoils the otherwise successful Smarr relation [4] and a remedy was proposed: to raise $\Lambda$ to the status of a thermodynamic variable, on a par with the temperature, while at the same time the black hole mass should be interpreted as the thermodynamic potential associated with the enthalpy, rather than the heretofore more usual interpretation of internal energy. It is then very natural to identify $\Lambda$ as being proportional to a pressure and the thermodynamic variable conjugate to the pressure can be interpreted as a volume for the black hole [5]. The idea of promoting $\Lambda$ to the status of a thermodynamic variable is not new, [6]-[11], but it is only recently that a volume has entered the picture in this context. For a rotating black hole in four dimensions this thermodynamic volume does not have an obvious relation to any geometric volume, though they agree if the black hole is not rotating, [12]. Nevertheless, with the volume included, there is a remarkable similarity between the black hole equation of state and that of a Van der Waals gas, [12]-[17].

We cannot vary $\Lambda$ in our Universe but we can nevertheless gain considerable conceptual insight into the nature of black hole thermodynamics by performing a gedanken experiment performing such a variation. Varying $\Lambda$ can have definite consequences: treating it as a thermodynamic variable, interpreted as a pressure, introduces a $P d V$ term into the first law of black hole thermodynamics which affects the efficiency of a Penrose process. In 4-dimensions, for example, the maximal efficiency for energy extraction from a rotating electrically neutral black hole is increased from $29 \%$ in asymptotically flat space-time to $52 \%$ in asymptotically anti-de Sitter space-time, while for an electrically charged black hole the corresponding figures are $50 \%$ and $75 \%,[12$. Of course we do not live in anti-de Sitter space, so this observation is unlikely to have observable consequences, just as the Hawking temperature has no observable consequences for astrophysical black holes. Nevertheless it is interesting conceptually to think about the possible consequences of such a variation. For example a negative cosmological constant can stabilise an otherwise thermodynamically unstable black hole, provided $|\Lambda|$ is of sufficient magnitude, leading to the well-known Hawking-Page transition [18]. Also in 
the AdS/CFT correspondence approach to condensed matter systems [19], a negative cosmological constant could generate a positive pressure in the condensed matter system, a pressure which in general we would wish to be able to vary.

Asymptotically anti-de Sitter space-time, in different dimensions, is also of central importance in the gauge gravity/correspondence [20] and its applications in non-abelian gauge theories. A consequence of this correspondence is a relation between the value of the cosmological constant (or, equivalently, the square of the anti-de Sitter curvature length $L$ ), the Planck length $l_{P}$ and the number of colours in the $S U(N)$ gauge theory, $\left(L / l_{P}\right)^{4} \approx N$, so varying $\Lambda$ is varying $N$, and $N \rightarrow \infty$ in the classical limit. As long as $N$ is very large there is nothing wrong with considering it to be a continuous variable (this is exactly how the number of particles in a gas is treated in the thermodynamic limit). Insights into the gauge/gravity correspondence might well be gained by considering the number of colours to be a thermodynamic variable.

An important physical quantity in any thermodynamics system that behaves like a gas is the compressibility: low compressibility implies a stiff equation of state and a stable system while a large compressibility implies a soft equation of state and a system which is verging on instability. Investigating the compressibility of a black hole solution therefore offers the possibility of learning something about stability without leaving the parametric framework of the solution.

The compressibility of rotating, asymptotically anti-de Sitter (AdS) black holes in 4-dimensions was investigated in [21, including asymptotically flat space-times as a limiting case. In that work it was shown that the thermodynamic compressibility of a rotating solar mass black hole in asymptotically flat space-time is some 4 orders of magnitude less the that of a neutron star of similar mass, sustained by degeneracy pressure.

In this paper the investigation of the compressibility of rotating black holes is extended to dimensions greater than four, where it is known that there are instabilities for ultra-spinning black holes. To that end we first derive the compressibility of a rotating asymptotically AdS Myers-Perry black hole in $D$ space-time dimensions. Our aim is to derive the compressibility and the speed of sound for asymptotically flat Myers-Perry black holes, but we must include a non-zero $\Lambda$ in order to obtain the volume and the compressibility before taking the limit $\Lambda \rightarrow 0$. In this limit the expressions simplify considerably and the compressibility and the speed of sound can be expressed rather compactly in terms of the quadratic and quartic Casimirs 
of $S O(D-1)$ associated with the angular momenta of the black hole.

As was emphasised in [12], it is crucial that one starts with rotating black holes: if there is no rotation the entropy $S$ and the volume $V$ are both functions of the event horizon radius $r_{h}$ only - they are not independent and cannot be considered to be independent thermodynamic variables they become independent only when the black hole rotates. This is not a pathology it is merely due to the fact the Legendre transform is not well defined in the limit of zero rotation. The enthalpy, $H(S, P, J)$, is linear in $P$ when $J=0$ and hence the Legendre transform is not invertible in this limit. Everything is well defined as $J \rightarrow 0$, provided one takes all derivatives and performs any desired Legendre transforms first before setting $J=0$. Setting $J=0$ at the start misses crucial aspects of the thermodynamics as far as the thermodynamic volume is concerned, but everything is consistent if the $J=0$ case is always approached by setting $J \neq 0$ first and only letting $J \rightarrow 0$ after any necessary manipulations involving Legendre transforms have been done.

It is well known that Kerr black holes are thermodynamically unstable, indeed all of the rotating black hole space-times considered here are thermodynamically unstable in the asymptotically flat limit, [22]. In $D=4$ the Schwarzschild black hole can be stabilised by placing it in a cavity with a heat bath at a finite distance from the horizon [23] or by switching on a negative cosmological constant [18] of sufficient magnitude. A negative cosmological constant can also stabilise rotating black holes in $D>4$ though, while it may be possible to stabilise rotating black holes by using a rotating cavity of finite size, it seems unlikely that one could ignore back-reaction and recover an asymptotically flat space-time if the radius of the cavity is sent to infinity. Nevertheless one can gain significant insight into black hole thermodynamics by analysing thermodynamically unstable black holes, indeed this is how Hawking made the discovery that black holes could evaporate. In this work we shall only consider isolated asymptotically AdS black holes (which can be stable if the magnitude of $\Lambda$ is large enough) and their asymptotically flat limits.

An issue that should be addressed in discussing black hole thermodynamics is the rôle of extensive versus intensive variables. In standard thermodynamics it is clear which variables scale with the volume of the system and which do not. In black hole thermodynamics, with $c=1$, all relevant thermodynamic variables can be made to scale with dimensions of length to some power by judicial use of Newton's constant. In $D$ space-time dimensions the 
key players for an electrically neutral black hole, which is the case for the analysis presented here, have the following length dimensions :

\begin{tabular}{|l|c|}
\hline Mass, $M$ (enthalpy, $H)$ & $D-3$ \\
Entropy, $S$ (area) & $D-2$ \\
Angular momenta, $J^{i}$ & $D-2$ \\
Volume, $V$ & $D-1$ \\
Temperature, $T$ & -1 \\
Angular velocity, $\Omega_{i}$ & -1 \\
Pressure, $P$ (Cosmological constant, $\Lambda$ ) & -2 \\
\hline
\end{tabular}

Identifying the mass with the enthalpy $M=H(S, J, P)$ gives

$$
d H=T d S+\Omega_{i} d J^{i}+V d P
$$

and scaling produces the Smarr relation

$$
(D-3) M=(D-2) S T+(D-2) \Omega_{i} J^{i}-2 P V .
$$

It is thus very natural to interpret the variables whose scaling depends on the space-time dimension $D$ as extensive and those whose dimension is independent of $D$ as intensive, and this will be the point of view adopted in this work. Thus $H, S, J^{i}$ and $V$ are considered to be extensive, while $T$, $\Omega_{i}$ and $P$ are intensive. The internal energy $U(S, J, V)=H-P V$ is thus a function of purely extensive variables, [12], while the enthalpy is not.

We restrict the analysis here to asymptotically AdS and asymptotically flat space-times. The thermodynamics of black holes in $\Lambda>0$ space-times is a notoriously delicate issue. First steps in understanding the rôle of a thermodynamic volume of black holes in this case were taken in [24] but unresolved issues remain, these are left for future work and are avoided here by restricting to $\Lambda \leq 0$.

In section 92 we summarise the relevant features of asymptotically AdS Myers-Perry black holes, determine the thermodynamic volume and describe the compressibility, the main result is the compressibility in equation (24). In $\$ 3$ the $\Lambda \rightarrow 0$, asymptotically flat, limit is investigated; the compressibility, given in (35) and speed of sound in (41), are derived and physical implications are discussed, particularly in relation to ultra-spinning black holes in $D>4$. The conclusions discuss some implications of the results and possible future directions. Finally some technical details are confined to two appendices. 


\section{AdS Myers-Perry black holes}

Rotating black holes in $D$-dimensions must be treated slightly differently for even and odd $D$ because the rotation group $S O(D-1)$, acting on the event horizon which is assumed to have the topology of a $(D-2)$-dimensional sphere, has different characterisations of angular momenta in the even and odd dimensional cases. The Cartan sub-algebra has dimension $\frac{D-2}{2}$ for even $D$ and $\frac{D-1}{2}$ for odd $D$ so a general state of rotation is specified by $\frac{D-2}{2}$ independent angular momenta in even $D$ and $\frac{D-1}{2}$ in odd $D$. Let $p=\left\lfloor\frac{D-1}{2}\right\rfloor$, the integral part of $\frac{D-1}{2}$, be the dimension of the Cartan sub-algebra of $S O(D-1)$, then there are $p$ independent angular momenta $J_{i}, i=1, \ldots, p$. It is notationally convenient to define $\epsilon=\frac{1+(-1)^{D}}{2}$, so $\epsilon=1$ for even $D$ and $\epsilon=0$ for odd $D$, and then

$$
p=\frac{D-1-\epsilon}{2}
$$

In this notation the unit $(D-2)$-dimensional sphere can be described in terms of Cartesian co-ordinates $x_{a}$ in $\mathbf{R}^{D-1}$ by

$$
\sum_{a=1}^{D-1} x_{a}^{2}=1,
$$

and we can write this as

$$
\sum_{i=1}^{p} \rho_{i}^{2}+\epsilon y^{2}=1
$$

where $x_{2 i-1}+i x_{2 i}=\rho_{i} e^{i \phi_{i}}, i=1, \ldots, p$, are complex co-ordinates for both the even and odd cases while $y=x_{D-1}$ is only necessary for even $D$. $\rho_{i}, \phi_{i}$ and $y$ are then (redundant) co-ordinates that can be used to parameterise the sphere and, for the black hole, $J_{i}$ are angular momenta in the $\left(x_{2 i-1}, x_{2 i}\right)$ plane.

The first rotating black solutions to Einstein's equations in dimension greater than four were were the asymptotically flat solutions of Myers and Perry [25]. Rotating black holes in 5-dimensions with a cosmological constant, $\Lambda$, were constructed in [26] and the generalisation to the $D$-dimensional metric was found in [27]: they are solutions of Einstein's equations with Ricci tensor 1

$$
R_{\mu \nu}=\frac{2 \Lambda}{(D-2)} g_{\mu \nu}
$$

\footnotetext{
${ }^{1}$ We use units with Newton's constant and the speed of light set to unity, $G=c^{2}=1$.
} 
We shall focus on $\Lambda \leq 0$ here, as the thermodynamics is then better understood, and for notational convenience we define

$$
\lambda=-\frac{2 \Lambda}{(D-1)(D-2)} \geq 0
$$

The line element in [27] can then be expressed, in Boyer-Linquist co-ordinates, ast

$$
\begin{aligned}
d s^{2}= & -W\left(1+\lambda r^{2}\right) d t^{2}+\frac{2 \mu}{U}\left(W d t-\sum_{i=1}^{p} \frac{a_{i} \rho_{i}^{2} d \phi_{i}}{1-\lambda a_{i}^{2}}\right)^{2} \\
& +\left(\frac{U}{Z-2 \mu}\right) d r^{2}+\epsilon r^{2} d y^{2}+\sum_{i=1}^{p}\left(\frac{r^{2}+a_{i}^{2}}{1-\lambda a_{i}^{2}}\right)\left(d \rho_{i}^{2}+\rho_{i}^{2} d \phi_{i}^{2}\right) \\
& -\frac{\lambda}{W\left(1+\lambda r^{2}\right)}\left(\sum_{i=1}^{p}\left(\frac{r^{2}+a_{i}^{2}}{1-\lambda a_{i}^{2}}\right) \rho_{i} d \rho_{i}+\epsilon r^{2} y d y\right)^{2},
\end{aligned}
$$

where the functions $W, Z$ and $U$ are

$$
\begin{aligned}
W & =\epsilon y^{2}+\sum_{i=1}^{p} \frac{\rho_{i}^{2}}{1-\lambda a_{i}^{2}} \\
Z & =\frac{\left(1+\lambda r^{2}\right)}{r^{2-\epsilon}} \prod_{i=1}^{p}\left(r^{2}+a_{i}^{2}\right) \\
U & =\frac{Z}{1+\lambda r^{2}}\left(1-\sum_{i=1}^{p} \frac{a_{i}^{2} \rho_{i}^{2}}{r^{2}+a_{i}^{2}}\right) .
\end{aligned}
$$

The $a_{i}$ are rotation parameters in the $\left(x_{2 i-1}, x_{2 i}\right)$-plane, restricted to $a_{i}^{2}<$ $1 / \lambda$, and $\mu$ is a mass parameter.

Many of the properties of the space-time with line element (9) were described in [27]. There is an event horizon at $r_{h}$, the largest root of $Z-2 \mu=0$, SO

$$
\mu=\frac{\left(1+\lambda r_{h}^{2}\right)}{2 r_{h}^{2-\epsilon}} \prod_{i=1}^{p}\left(r_{h}^{2}+a_{i}^{2}\right)
$$

with area

$$
\mathcal{A}_{h}=\frac{\varpi}{r_{h}^{1-\epsilon}} \prod_{i=1}^{p} \frac{r_{h}^{2}+a_{i}^{2}}{1-\lambda a_{i}^{2}},
$$

\footnotetext{
${ }^{2}$ The form given here differs slightly from that in [27] in that our ordinates, $t$ and $\phi_{i}$, are related to those of [27, $\tau$ and $\varphi_{i}$, by $d \tau=d t$ and $d \phi_{i}=d \varphi_{i}-\lambda a_{i} d t$.
} 
where $\varpi$ is is the volume of the round unit $(D-2)$-sphere,

$$
\varpi=\frac{2 \pi^{\frac{(D-1)}{2}}}{\Gamma\left(\frac{D-1}{2}\right)} .
$$

The Bekenstein-Hawking entropy is

$$
S=\frac{\varpi}{4 r_{h}^{1-\epsilon}} \prod_{i=1}^{p} \frac{r_{h}^{2}+a_{i}^{2}}{1-\lambda a_{i}^{2}}
$$

and the Hawking temperature is, with $\hbar=1$,

$$
T=\frac{r_{h}}{2 \pi}\left(1+\lambda r_{h}^{2}\right) \sum_{i=1}^{p} \frac{1}{r_{h}^{2}+a_{i}^{2}}+\frac{(2-\epsilon)\left(\epsilon \lambda r_{h}^{2}-1\right)}{4 \pi r_{h}} .
$$

The angular momenta and the ADM mass, $M$, of the black hole are related to the metric parameters via

$$
\begin{aligned}
J_{i} & =\frac{\mu \varpi a_{i}}{4 \pi\left(1-\lambda a_{i}^{2}\right) \prod_{j}\left(1-\lambda a_{j}^{2}\right)}, \\
M & =\frac{\mu \varpi}{8 \pi \prod_{j}\left(1-\lambda a_{j}^{2}\right)}\left(D-2+2 \lambda \sum_{i=1}^{p} \frac{a_{i}^{2}}{1-\lambda a_{i}^{2}}\right) \\
& =\frac{(D-2) \mu \varpi}{8 \pi \prod_{j}\left(1-\lambda a_{j}^{2}\right)}+\lambda \sum_{i=1}^{p} J_{i} a_{i},
\end{aligned}
$$

while the angular velocities are

$$
\Omega_{i}=\frac{\left(1+\lambda r_{h}^{2}\right) a_{i}}{\left(r_{h}^{2}+a_{i}^{2}\right)}
$$

It was argued in [3] that, in the presence of a cosmological constant, the correct thermodynamic interpretation of the black hole mass is that it is the enthalpy of the system

$$
M=H(S, P, J),
$$

where $J$ stands for all the $J_{i}$ collectively, and the pressure is

$$
P=-\frac{\Lambda}{8 \pi}=\frac{(D-1)(D-2) \lambda}{16 \pi} .
$$


The thermodynamic volume, $V$, is defined as the variable thermodynamically conjugate to $P[\underline{5}, 12$,

$$
V=\left.\frac{\partial M}{\partial P}\right|_{S, J}=\left.\frac{16 \pi}{(D-1)(D-2)} \frac{\partial M}{\partial \lambda}\right|_{S, J} .
$$

Details of the calculation of the thermodynamic volume by this technique are given in appendix $\mathrm{A}$ and here we quote the result (46)

$$
\begin{aligned}
V & =\frac{r_{h} \mathcal{A}_{h}}{D-1}\left\{1+\frac{\left(1+\lambda r_{h}^{2}\right)}{(D-2) r_{h}^{2}} \sum_{i=1}^{p} \frac{a_{i}^{2}}{\left(1-\lambda a_{i}^{2}\right)}\right\} \\
& =\frac{r_{h} \mathcal{A}_{h}}{D-1}+\frac{8 \pi}{(D-2)(D-1)} \sum_{i=1}^{p} a_{i} J_{i} .
\end{aligned}
$$

With the substitution $\lambda \rightarrow g^{2}$ this agrees with the result [28] for the black hole volume, derived from the assumption that the Smarr relation,

$$
(D-3) M=(D-2) T S+(D-2) \sum_{i=1}^{p} \Omega_{i} J_{i}-2 P V
$$

holds. For $D=4$ it reproduces the corresponding expression in [12]. With the substitution $\lambda \rightarrow-g^{2}$, (22) agrees with the black hole thermodynamic volume quoted in [24] for $\Lambda>0$, again determined by assuming the Smarr relation holds, but avoiding the complication of the existence of the cosmological horizon that is present in this case.

It is now possible to define the adiabatic compressibility of the black hole [21] as

$$
\kappa=-\left.\frac{1}{V} \frac{\partial V}{\partial P}\right|_{S, J}
$$

With the explicit form of the thermodynamic volume in (22) the compressibility can be computed using the technique outlined in appendix B; it evaluates to

$$
\kappa=\frac{16 \pi\left(1+\lambda r_{h}^{2}\right)}{(D-1)(D-2)^{2}} \frac{\left\{\sum_{i=1}^{p} \frac{a_{i}^{4}}{1-\lambda^{2} a_{i}^{4}}-\frac{1}{(D-2)}\left(\sum_{i=1}^{p} \frac{a_{i}^{2}}{1-\lambda a_{i}^{2}}\right)\left(\sum_{i=1}^{p} \frac{a_{i}^{2}}{1+\lambda a_{i}^{2}}\right)\right\}}{\left\{r_{h}^{2}+\frac{\left(1+\lambda r_{h}^{2}\right)}{(D-2)} \sum_{i=1}^{p} \frac{a_{i}^{2}}{1-\lambda a_{i}^{2}}\right\}\left\{1-\frac{2 \lambda}{(D-2)} \sum_{i=1}^{p} \frac{a_{i}^{2}}{1+\lambda a_{i}^{2}}\right\}} .
$$


It is shown in the appendix that $\kappa \geq 0$. It is also not difficult to prove that it is bounded above for $\lambda>0$ : to see this first observe that the denominator never vanishes because

$$
D-2-2 \lambda \sum_{i=1}^{p} \frac{a_{i}^{2}}{1+\lambda a_{i}^{2}}=\epsilon-1+2 \sum_{i=1}^{p} \frac{1}{1+\lambda a_{i}^{2}} \geq \frac{D-3-\epsilon}{2},
$$

with equality when all the $a_{i}$ achieve the maximum value, $a_{1}^{2}=\cdots a_{p}^{2}=\frac{1}{\lambda}$. The numerator can diverge though, if any or all of the $a_{i}^{2}$ approach $\frac{1}{\lambda}$, but when this happens the first factor in curly brackets in the denominator also diverges, the singularities cancel and $\kappa$ remains finite. For example, if $m$ of the $a_{i}^{2}$ approach $1 / \lambda$, with $1 \leq m \leq p$, and the others are all zero, then

$$
\kappa \rightarrow \frac{8 \pi}{(D-1)(D-2) \lambda}=\frac{1}{2 P}
$$

reflecting the fact that $V \propto \frac{1}{\sqrt{P}}$ in this limit.

A thermodynamic speed of sound, $c_{s}$, can be defined by using the homogeneous density,

$$
\rho=\frac{M}{V}=\frac{(D-1)(D-2)\left(1+\lambda r_{h}^{2}\right)}{16 \pi r_{h}^{2}} \frac{\left(1+\frac{2 \lambda}{D-2} \sum_{i=1}^{p} \frac{a_{i}^{2}}{1-\lambda a_{i}^{2}}\right)}{\left(1+\frac{\left(1+\lambda r_{h}^{2}\right)}{(D-2) r_{h}^{2}} \sum_{i=1}^{p} \frac{a_{i}^{2}}{1-\lambda a_{i}^{2}}\right)}
$$

The usual thermodynamic relation can then be used to obtain a speed of sound,

$$
\frac{1}{c_{s}^{2}}=\left.\frac{\partial \rho}{\partial P}\right|_{S, J}=1+\rho \kappa \geq 1
$$

so $0 \leq c_{s}^{2} \leq 1$. Again, for example, taking $m$ of the $a_{i}^{2}$ to approach $1 / \lambda$ with the others all zero,

$$
\rho \rightarrow \frac{(D-1)(D-2) \lambda}{8 \pi},
$$

and $\rho \kappa \rightarrow 1$, giving $c_{s}^{2}=\frac{1}{2}$. 


\section{$3 \quad$ Isentropic processes in asymptotically flat Myers-Perry space-times}

When the cosmological constant vanishes many of the expressions in the previous section simplify considerably. In particular

$$
\begin{aligned}
& M=\frac{(D-2) \varpi \mu}{8 \pi}, \quad S=\frac{\varpi}{4 r^{1-\epsilon}} \prod_{i=1}^{p}\left(r_{h}^{2}+a_{i}^{2}\right)=\frac{4 \pi}{D-2} M r_{h} \\
& J_{i}=\frac{2 M a_{i}}{D-2}, \quad \Omega_{i}=\frac{a_{i}}{r_{h}^{2}+a_{i}^{2}} .
\end{aligned}
$$

In this section we shall focus on isentropic processes, for which it is convenient to define the dimensionless angular momenta

$$
\mathcal{J}_{i}=\frac{2 \pi J_{i}}{S}=\frac{a_{i}}{r_{h}}
$$

in terms of which the mass is

$$
M=\frac{(D-2)}{16 \pi} \varpi r_{h}^{D-3} \prod_{i=1}^{p}\left(1+\mathcal{J}_{i}^{2}\right)
$$

and the entropy is

$$
S=\frac{\varpi}{4} r_{h}^{D-2} \prod_{i=1}^{p}\left(1+\mathcal{J}_{i}^{2}\right) .
$$

The thermodynamic volume is

$$
V=\frac{r_{h} \mathcal{A}_{h}}{D-1}\left(1+\frac{\sum_{i} \mathcal{J}_{i}^{2}}{D-2}\right)=V_{0} \prod_{i=1}^{p}\left(1+\mathcal{J}_{i}^{2}\right)\left(1+\frac{\sum_{i} \mathcal{J}_{i}^{2}}{D-2}\right),
$$

where $V_{0}=\frac{\varpi r_{h}^{D-1}}{D-1}$ is the volume of an ordinary $D-1$ dimensional sphere of radius $r_{h}$.

The $\lambda \rightarrow 0$ limit of (24) is finite:

$$
\kappa=\frac{16 \pi r_{h}^{2}}{(D-1)(D-2)^{2}}\left\{\frac{(D-2) \sum_{i} \mathcal{J}_{i}^{4}-\left(\sum_{i} \mathcal{J}_{i}^{2}\right)^{2}}{D-2+\sum_{i} \mathcal{J}_{i}^{2}}\right\},
$$

with

$$
r_{h}^{2}=\left\{\frac{4 S}{\varpi \prod_{i}\left(1+\mathcal{J}_{i}^{2}\right)}\right\}^{\frac{2}{D-2}}
$$


Thus at fixed $S$ the compressibility is simply expressible entirely in terms of quadratic and quartic Casimirs of $S O(D-1)$.

For $D \geq 4, \kappa$ is positive, and it is identically zero in $D=3$ : this latter result is in intuitive accord with the fact that gravity has no dynamics in the bulk in 3-dimensions, all of the interesting physics is in the boundary conditions.

To understand $\kappa$ fully, it is necessary to take account of the constraints imposed by the condition that $T \geq 0$. For $\lambda=0$

$$
T=\frac{1}{2 \pi r_{h}}\left(\sum_{i=1}^{p} \frac{1}{1+\mathcal{J}_{i}^{2}}-1+\frac{\epsilon}{2}\right) \quad \Rightarrow \quad \sum_{i=1}^{p} \frac{1}{1+\mathcal{J}_{i}^{2}} \geq 1-\frac{\epsilon}{2} .
$$

For $D>4$ it is possible for some of the $\mathcal{J}_{i}$ to tend to infinity, but not all of them - the well known phenomenon of ultra-spinning black holes 3

Equation (37) says that the locus of allowed temperatures is thus bounded by hyperbolae in $\mathcal{J}$-space. The case for $D=6$ is plotted in figure 1 below. This is very similar to plots in [29], except there the $J_{i}$ are normalised using the appropriate power of the mass, relevant for isenthalpic processes, while here the entropy is used, for isentropic processes.

When all the $\mathcal{J}_{i}$ are small the compressibility is small and the equation of state is very stiff, the black hole is completely incompressible for $\mathcal{J}_{i}=0$. However the compressibility can diverge if some $\mathcal{J}_{i}$ are kept small while others are sent to infinity. For example, if $\mathcal{J}_{1}=\cdots=\mathcal{J}_{p-m}=0$ and $\mathcal{J}_{p-m+1}=\cdots \mathcal{J}_{p}=L$, then $T \geq 0$ for $L \rightarrow \infty$ provided $m \leq \frac{D-3}{2}$. Also (36) implies that $r_{h}^{2} \propto L^{-\frac{4 m}{D-2}}$ so

$$
\kappa \sim L^{\frac{2(D-2 m-2)}{D-2}},
$$

\footnotetext{
${ }^{3}$ Note that, although an ultra-spinning black hole has a large $J_{i}$, the corresponding angular velocity need not be large: indeed $\Omega_{i} \rightarrow 0$ as $J_{i} \rightarrow \infty$. The inverse of the isentropic momentum of inertia tensor is

$$
\mathcal{I}_{i j}^{-1}=\left.\frac{\partial \Omega_{i}}{\partial J_{j}}\right|_{S}=\frac{1}{M r_{h}^{2}}\left\{\frac{(D-2)}{2} \frac{\left(1-\mathcal{J}_{i}^{2}\right)}{\left(1+\mathcal{J}_{i}^{2}\right)^{2}} \delta_{i j}+\frac{\mathcal{J}_{i} \mathcal{J}_{j}}{\left(1+\mathcal{J}_{i}^{2}\right)\left(1+\mathcal{J}_{j}^{2}\right)}\right\} .
$$

For large $\mathcal{J}_{i}, \mathcal{I}^{-1}$ develops a negative eigenvalue and a negative moment of inertia implies that $\Omega_{i}$ decreases as $J_{i}$ increases. Indeed if one of the $\mathcal{J}_{i}$ tends to infinity as $\mathcal{J}_{i}=L \rightarrow \infty$, at constant finite $S$, then $r_{h} \approx L^{-\frac{2}{D-2}}$, from (33), and the corresponding element of $\mathcal{I}^{-1} \approx-L^{\frac{4}{D-2}-2} \rightarrow 0$ as $L \rightarrow \infty$, provided $D>4$. Ultra-spinning black holes do not have large angular momenta because they have large angular velocity, they have large angular momenta because their moment of inertia diverges as $J_{i} \rightarrow \infty$.
} 


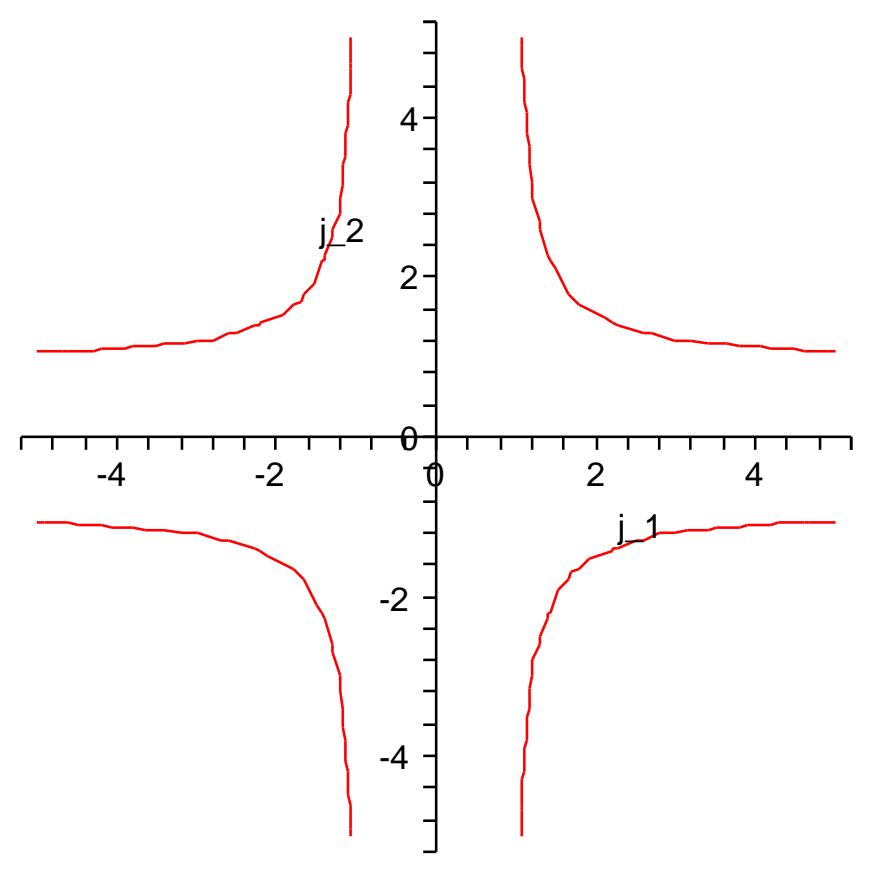

Figure 1: The locus of extremal black holes, $T=0$, for $D=6$. $T>0$ requires the angular momenta to lie inside the region bounded by the hyperbolae.

which diverges if $m<\frac{D-2}{2}$, so $\kappa$ diverges for $1 \leq m \leq \frac{D-3}{2}$ with this configuration of angular momenta. The divergence is fastest for $m=1$.

When the compressibility becomes large the black hole equation of state is very soft. For example the compressibility for $D=6$ is plotted in figure 2 and it grows indefinitely for large angular momenta along either the $\mathcal{J}_{1}$ or the $\mathcal{J}_{2}$ axis, i.e. $m=1$.

It was suggested in 30] that ultra-spinning black holes should be dynamically unstable for large angular momentum, and subsequent numerical and analytical work supports this proposal [31]-339. Large compressibility can be taken as a sign of an instability setting in, although there is no indication in equation (35) of a boundary in $\mathcal{J}$-space were a dynamical instability might manifest itself, the expression for the compressibility implies that the instability sets in more quickly when only one angular momentum is taken 


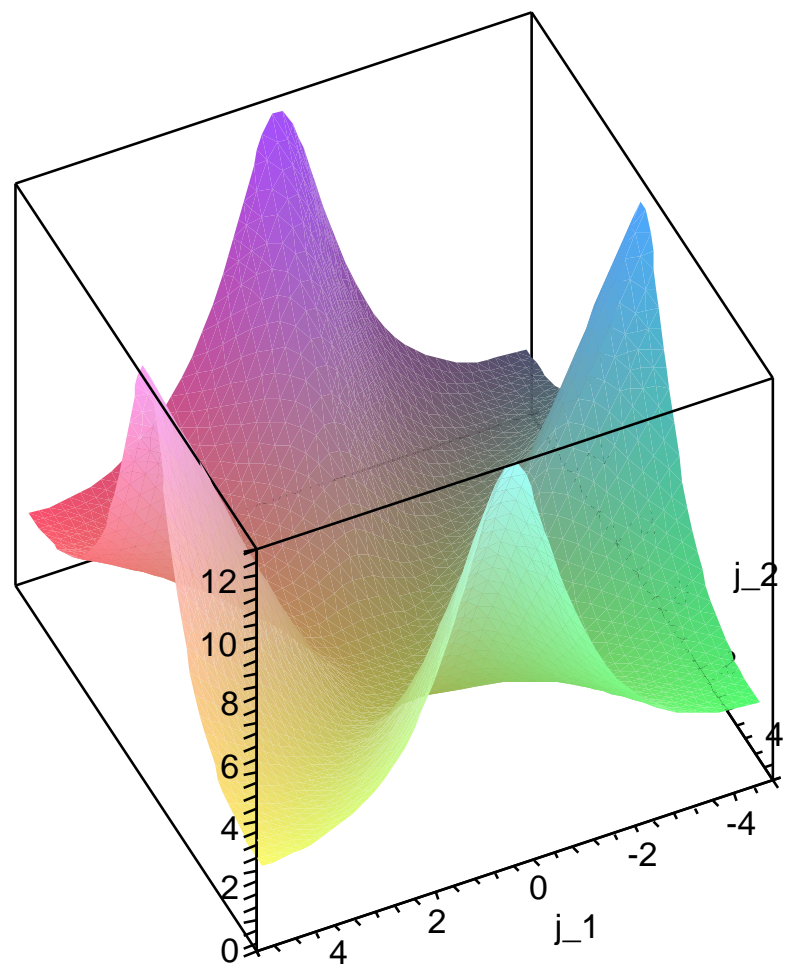

Figure 2: The compressibility of a black hole in $\mathrm{D}=6$ as a function of $\mathcal{J}_{1}$ and $\mathcal{J}_{2}$.

to be large compatible with the pancake structure of [31].

Again a thermodynamic speed of sound can be defined using

$$
\rho=\frac{(D-1)(D-2)^{2}}{16 \pi r_{h}^{2}} \frac{1}{\left(D-2+\sum_{i=1}^{p} \mathcal{J}_{i}^{2}\right)}
$$

which gives, with (35) in (28),

$$
c_{s}^{2}=\frac{1}{(D-2)} \frac{\left(D-2+\sum_{i} \mathcal{J}_{i}^{2}\right)^{2}}{\left(D-2+2 \sum_{i} \mathcal{J}_{i}^{2}+\sum_{i} \mathcal{J}_{i}^{4}\right)} .
$$

It is not immediately clear how the thermodynamic speed of sound might be related to a fluid dynamical speed of sound, but it is noteworthy that the thermodynamic speed of sound is least when the compressibility is greatest, 
as one would expect for a soft equation of state. Indeed $\frac{1}{D-2} \leq c_{s}^{2} \leq 1$ with $c_{s}=1$ for $\mathcal{J}_{i}=0$ and $c_{s}^{2} \rightarrow \frac{1}{D-2}$ as any one $\mathcal{J}_{i} \rightarrow \infty$ with all others remaining finite. It is possible that the thermodynamic speed of sound is related to the velocity of the kind of waves and vibrations envisaged in 31. associated with the instability of an ultra-spinning black hole, at least for $D>4$.

\section{Conclusions}

A cosmological constant spoils the Smarr relation for black hole thermodynamics unless it is given the status of a thermodynamic variable, most naturally interpreted as proportional to a pressure. A consistent interpretation of the ADM mass of the black hole, in terms of thermodynamic potentials, is that it is the enthalpy of the black hole. A thermodynamic volume can then be defined as being the thermodynamic variable conjugate to the pressure, in terms of Legendre transforms.

The main results are the thermodynamic volume (22), computed explicitly as the Legendre transform variable conjugate of the pressure rather than by assuming the Smarr relation, and the compressibility (24) for Myers-Perry black holes in asymptotically anti-de Sitter, $D$-dimensional, space-time. The corresponding expressions for asymptotically flat space-times then follow easily from the $\Lambda \rightarrow 0$ limit, and the corresponding quantities for asymptotically flat Myers-Perry black holes are given in equations (22) and (35) respectively. In addition the speed of sound can be expressed in terms of Casimirs of the rotation group, $S O(D-1)$, and is given in (41).

We emphasise again that it is crucial that the black hole is rotating. It is clear from equations (11), (13) and (22) that, when all $a_{i} \rightarrow 0$, the entropy $S\left(r_{h}, \lambda, a_{i}\right)$ and the volume $V\left(r_{h}, \lambda, a_{i}\right)$ are both functions of the event horizon radius $r_{h}$ only, we then have $V\left(r_{h}\right)$ and $S\left(r_{h}\right)$ and $V$ can be written uniquely as a function of the single variable $S$ : they cannot be considered to be independent thermodynamic variables in this limit. The volume is an independent thermodynamic variable only when the black hole rotates, otherwise the Legendre transform is not well defined, as was first pointed out in [12]. This is reflected in the fact that the isentropic compressibility (24) vanishes as $\mathcal{J}_{i} \rightarrow 0$ : fixing $S$ fixes $V$ when the black hole is non-rotating, hence it is incompressible.

The discussion here has been restricted to electrically neutral rotating black holes, leaving open the question of how electric charge might affect 
compressibility.

It would be very interesting to develop these ideas in the context of positive $\Lambda$, but we immediately hit the problem of having two horizons to contend with, a black hole horizon and a cosmological horizon, leading to two, in general different, temperatures and raising the question of how to define thermodynamic potentials for such a system. A preliminary discussion of thermodynamic volumes in this context was given in [24, but only by treating the two horizons as essentially independent and defining two independent volumes. The volume associated with the black hole horizon in [24] was the same as (22), but with $\lambda=-g^{2}$ negative, and it is perhaps significant in this context that $\kappa$ in (24) remains positive under this continuation to negative $\lambda$, provided $r_{h}^{2}<-\frac{1}{\lambda}$. However a completely consistent integrated thermodynamic treatment of asymptotically de Sitter space-times still eludes us.

\section{A Thermodynamic volume}

The thermodynamic volume is calculated by differentiating the mass (16) with respect to $\lambda$, keeping the entropy and the angular momenta fixed. To this end we note that (10), (13), (15) and (16) allow us to write

$$
J_{i}=\frac{S}{2 \pi r_{h}} \frac{\left(1+\lambda r_{h}^{2}\right)}{\left(1-\lambda a_{i}^{2}\right)} a_{i}
$$

and demanding $\left.d J_{i}\right|_{S}=0$ then gives

$$
d a_{i}=\frac{a_{i}}{\left(1+\lambda a_{i}^{2}\right)\left(1+\lambda r_{h}^{2}\right)}\left\{\left(1-\lambda r_{h}^{2}\right)\left(1-\lambda a_{i}^{2}\right) \frac{d r_{h}}{r_{h}}-\left(r_{h}^{2}+a_{i}^{2}\right) d \lambda\right\} .
$$

A second relation between $d a_{i}, d r_{h}$ and $d \lambda$ follows from $\left.d S\right|_{J_{i}}=0$ in (13), allowing the elimination of $d a_{i}$ to give

$$
d r_{h}=\left(\frac{\sum_{i} \frac{a_{i}^{2}}{1+\lambda a_{i}^{2}}}{D-2+2 \lambda \sum_{i} \frac{a_{i}^{2}}{1+\lambda a_{i}^{2}}}\right) r_{h} d \lambda,
$$

and we have all the ingredients necessary to calculate $\left.\frac{\partial}{\partial \lambda}\right|_{S, J}$ acting on any function of $\lambda, r_{h}$ and $a_{i}$. 
The thermodynamic volume is perhaps most easily calculated by combining (10), (13), and the mass in (16), to write

$$
M=\frac{S}{4 \pi} \frac{\left(1+\lambda r_{h}^{2}\right)}{r_{h}}\left(D-2+2 \lambda \sum_{i=1}^{p} \frac{a_{i}^{2}}{1-\lambda a_{i}^{2}}\right) .
$$

Using this equation (43) and (44) yields the following formula for the volume

$$
\begin{aligned}
V=\left.\frac{16 \pi}{(D-1)(D-2)} \frac{\partial M}{\partial \lambda}\right|_{S, J} & =\frac{4 r_{h} S}{D-1}\left\{1+\frac{\left(1+\lambda r_{h}^{2}\right)}{(D-2) r_{h}^{2}} \sum_{i=1}^{p} \frac{a_{i}^{2}}{\left(1-\lambda a_{i}^{2}\right)}\right\} \\
& =\frac{4 r_{h} S}{D-1}+\frac{8 \pi}{(D-2)(D-1)} \sum_{i=1}^{p} a_{i} J_{i} .
\end{aligned}
$$

\section{B Compressibility}

The compressibility can be evaluated by pushing the analysis of appendix A one step further and calculating

$$
\kappa=-\left.\frac{16 \pi}{(D-1)(D-2)} \frac{1}{V} \frac{\partial V}{\partial \lambda}\right|_{S, J}
$$

A tedious, but straightforward calculation, gives

$$
\kappa=\frac{16 \pi\left(1+\lambda r_{h}^{2}\right)}{(D-1)(D-2)^{2}} \frac{\left\{\sum_{i=1}^{p} \frac{a_{i}^{4}}{1-\lambda^{2} a_{i}^{4}}-\frac{1}{(D-2)}\left(\sum_{i=1}^{p} \frac{a_{i}^{2}}{1-\lambda a_{i}^{2}}\right)\left(\sum_{i=1}^{p} \frac{a_{i}^{2}}{1+\lambda a_{i}^{2}}\right)\right\}}{\left\{r_{h}^{2}+\frac{\left(1+\lambda r_{h}^{2}\right)}{(D-2)} \sum_{i=1}^{p} \frac{a_{i}^{2}}{1-\lambda a_{i}^{2}}\right\}\left\{1-\frac{2 \lambda}{(D-2)} \sum_{i=1}^{p} \frac{a_{i}^{2}}{1+\lambda a_{i}^{2}}\right\}} .
$$

We can show that $\kappa \geq 0$. First note that

$$
D-2-2 \lambda \sum_{i=1}^{p} \frac{a_{i}^{2}}{1+\lambda a_{i}^{2}}=\epsilon-1+2 \sum_{i=1}^{p} \frac{1}{1+\lambda a_{i}^{2}}>0
$$

hence both factors in curly brackets in the denominator of (48) are positive. It remains to show that the curly bracket in the numerator is positive. To this end define

$$
X_{i}^{ \pm}=\frac{a_{i}^{2}}{1 \pm \lambda a_{i}^{2}}
$$

and express the curly bracket in the numerator in terms of the bi-linear form,

$$
X^{+} . X^{-}:=\frac{1}{(D-2)} \sum_{i, j=1}^{p} X_{i}^{+} K_{i j} X_{j}^{-}
$$


$K_{i j}$ here are the components of the $p \times p$ matrix

$$
\mathbf{K}=(D-2) 1-\mathbf{I}
$$

where $\mathbf{I}$ is the $p \times p$ all of whose entries are 1 . The eigenvectors of $\mathbf{K}$ are the same as the eigenvectors of $\mathbf{I}$ and the latter has one eigenvalue equal to $p$ and $p-1$ degenerate zero eigenvalues, hence $\mathbf{K}$ has one eigenvalue equal to $D-2-p=\frac{D-3+\epsilon}{2}$ and $p-1$ eigenvalues equal to $D-2$. All that concerns us here is that $\mathbf{K}$ is positive definite. We can use the identity

$$
X^{+} \cdot X^{-}=\frac{1}{2}\left(\left(X^{+}+X^{-}\right) \cdot\left(X^{+}+X^{-}\right)-X^{+} \cdot X^{+}-X^{-} \cdot X^{-}\right),
$$

with $X_{i}^{+}+X_{i}^{-}=\frac{2 a_{i}^{2}}{1-\lambda^{2} a_{i}^{4}}$, to arrive at

$$
\begin{aligned}
X^{+} . X^{-} & =\sum_{i, j=1}^{p} \frac{\left(1-\lambda^{2} a_{i}^{2} a_{j}^{2}\right) a_{i}^{2} a_{j}^{2}}{\left(1-\lambda^{2} a_{i}^{4}\right)\left(1-\lambda^{2} a_{j}^{4}\right)} K_{i j} \\
& \geq\left(1-\lambda^{2} a_{\text {Max }}^{2}\right) \sum_{i, j=1}^{p} \frac{a_{i}^{2}}{\left(1-\lambda^{2} a_{i}^{4}\right)} K_{i j} \frac{a_{j}^{2}}{\left(1-\lambda^{2} a_{j}^{4}\right)},
\end{aligned}
$$

where $a_{\text {Max }}^{2}=\max \left(a_{1}^{2}, \ldots a_{p}^{2}\right)$. Since all $a_{i}$ satisfy $a_{i}^{2} \leq \frac{1}{\lambda}$ we have $1-\lambda^{2} a_{\text {Max }}^{2} \geq$ 0 and hence $X^{+} . X^{-} \geq 0$. The compressibility is thus bounded from below.

\section{References}

[1] J.D. Bekenstein, Lett. Nuovo. Cimento 4 (1972) 737;

J.D. Bekenstein, Phys. Rev. D7 (1973) 2333.

[2] S.W. Hawking, Nature 248 (1974) 30;

S.W. Hawking, Comm. Math. Phys. 43 (1975) 199;

S.W. Hawking, Phys. Rev. D13 (1976) 191.

[3] D. Kastor, S. Ray and J. Traschen, Class.Quant.Grav. 26 (2009) 195011, arXiv:0904.2765].

[4] L. Smarr, Phys. Rev. Lett. 30 (1973) 71.

[5] B.P. Dolan, Class. Quantum Grav. 28 (2011) 125020, arXiv:1008.5023]. 
[6] M. Henneaux and C. Teitelboim, Phys. Lett. 143B (1984) 415;

M. Henneaux and C. Teitelboim, Phys. Lett. 222B (1989) 195;

C. Teitelboim, Phys. Lett. 158B (1985) 293.

[7] Y. Sekiwa, Phys. Rev. D73 (2006) 084009, arXiv:hep-th/0602269.

[8] E.A. Larrañaga Rubio, arXiv:0711.0012 [gr-qc]].

[9] S. Wang, S. Wu, F. Xie and L. Dan, Chin. Phys. Lett. 23 (2006) 1096, arXiv:hep-th/0601147]; S. Wang, arXiv:gr-qc/0606109].

[10] M. Urano, A. Tomimatsu and H. Saida, Class. Quantum Grav. 26 (2009) 105010, arXiv:0903.4230 [gr-qc].

[11] H. Lu, Y. Pang, C.N. Pope and J.F. Vazquez-Poritz, Phys. Rev. D86 (2012) 044011, arXiv:1204.1062.

[12] B.P. Dolan, Class. Quantum Grav. 28 (2011) 235017, arXiv:1106.6260.

[13] D. Kubizn̆ák and B. Mann, JHEP 1207 (2012) 033, [1205.0559[hep-th]].

[14] S. Gunasekaran, D. Kubizn̆ák and R.B. Mann, JHEP 1211 (2012) 110, [1208.6251 [hep-th]].

[15] B.P. Dolan, in Open Questions in Cosmology, ed. G.J. Olmo (2012) InTech, arXiv:1209.1272.

[16] N. Altamirano, D. Kubizn̆ák and R.B. Mann, Re-entrant Phase Transitions in Rotating AdS Black Holes, arXiv:1306.5756[hep-th]].

[17] N. Altamirano, D. Kubizn̆ák, R.B. Mann and Z. Sherkatghanad, KerrAdS analogue of tri-critical point and solid/liquid/gas phase transition, arXiv:1308.2672 [hep-th]].

[18] S.W. Hawking and D.N. Page, Comm. Math. Phys. 87 (1983) 577.

[19] S.S. Gubser Phys. Rev. D78 (2008) 065034, arXiv:0801.2977].

[20] O. Aharony, S.S. Gubser, J. Maldacena, H. Ooguri and Y. Oz, Phys. Rep. 323 (2000) 183, hep-th/9905111.

[21] B.P. Dolan, Phys. Rev. D84 (2011) 127503, arXiv:1109.0198]. 
[22] O.J.C. Dias, P. Figueras, R. Monteiro, H.S. Reall and J.E. Santos, JHEP 1005 (2010) 076, arXiv:1001.4527 [hep-th]].

[23] J.W. York Jr., Phys. Rev. D 33 (1986) 2092.

[24] B.P. Dolan, D. Kastor, D. Kubizňák, R.B. Mann and J. Traschen, Phys. Rev. D87 (2013) 104017, arXiv:1301.5926].

[25] R.C. Myers and M.J. Perry, Annals Phys. 172 (1986) 304.

[26] S.W. Hawking, C.J. Hunter and M.M. Taylor-Roqbinson, Phys. Rev. D59 (1999) 064005, hep-th/9811056.

[27] G.W. Gibbons, H. Lü, D.N. Page and C.N. Pope, Phys. Rev. Lett. 93 (2004) 171102, hep-th/0409155];

G.W. Gibbons, H. Lü, D.N. Page and C.N. Pope, Geom. Phys. 53 (2005) 49, hep-th/0404008.

[28] M. Cvetic, G.W. Gibbons, D. Kubiznák and C.N. Pope, Phys. Rev. D84 (2011) 024037, arXiv:1012.2888.

[29] R. Emparan and H.S. Reall, Black Holes in Higher Dimensions, Living Rev. Rel. 11:6 (2008), arXiv:0801.3471].

[30] R. Emparan and R. Myers, JHEP 0309 (2003) 025, hep-th/0308056].

[31] R. Emparan, T. Harmark, V. Niarchos, N.A. Obers and M.J. Rodriguez, JHEP 0710 (2007). 110 [arXiv:0708.2181 [hep-th]].

[32] O.J.C. Dias, P. Figueras, R. Monteiro, J.E. Santos and R. Emparan, Phys. Rev. D80 (2009) 111701, arXiv:0907.2248 [hep-th]].

[33] D. Astefanesei, R.B. Mann, M. J. Rodriguez and C. Stelea, Class. Quantum Grav. 27 (2010) 165004, arXiv:0909.3852[hep-th]].

[34] M. Shibata and H. Yoshino, Phys. Rev. D81 (2010) 021501, arXiv:0912.3606[gr-qc]].

[35] Thermodynamic instability of doubly spinning black objects, D. Astefanesei, M.J. Rodriguez and S. Theisen arXiv:1003.2421 [hep-th]].

[36] M. Shibata and H. Yoshino, Phys. Rev. D81 (2010) 104035, arXiv:1004.4970 [gr-qc]]. 
[37] O.J.C. Dias, P. Figueras, R. Monteiro and J.E. Santos, Phys. Rev. D82 (2010) 104025. [arXiv:1006.1904[hep-th]].

[38] O.J.C. Dias, R. Monteiro and J.E. Santos, JHEP 1108 (2011) 139, arXiv:1106.4554 [hep-th]].

[39] R. Monteiro, M.J. Perry and J.E. Santos, Phys. Rev. D80 (2009) 024041, arXiv:0903.3256 [gr-qc]]. 